\title{
Gastrointestinal Manifestations in Children with Primary Immunodeficiencies: Single Center: 12 Years Experience
}

\author{
Bilge S. Akkelle ${ }^{\mathrm{a}}$ Engin Tutar ${ }^{\mathrm{a}}$ Burcu Volkan ${ }^{\mathrm{a}}$ Ozlem K. Sengul ${ }^{\mathrm{a}} \quad$ Ahmet Ozen $^{\mathrm{b}}$ \\ Cigdem A. Celikel ${ }^{c}$ Deniz Ertem ${ }^{\mathrm{a}}$ \\ ${ }^{a}$ Division of Pediatric Gastroenterology, Hepatology and Nutrition, Marmara University School of Medicine, Istanbul,

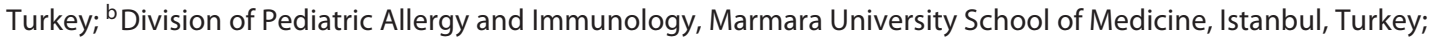 \\ 'Department of Pathology, Marmara University School of Medicine, Istanbul, Turkey
}

\author{
Keywords \\ Child · Endoscopy · Gut · Histopathology · Primary \\ immunodeficiency
}

\begin{abstract}
Background: It has been reported that $5-50 \%$ of patients with primary immune deficiencies (PID) may present with or develop gastrointestinal (GI) manifestations. Objective: This study was aimed at analyzing $\mathrm{Gl}$ and related endoscopic, histopathological findings in children with PID. Methods: Children with PID who were evaluated by endoscopy between 2005 and 2016 were enrolled in this study. Demographic data, growth parameters, signs and symptoms at diagnosis were obtained. Results: Of 425 children with PID, 195 had GI manifestations. Forty-seven of 195 children required endoscopic investigation, 30 (63.8\%) were male, and the mean age was $7.7 \pm 5$ years. The rate of consanguinity was $61.7 \%$, and the most common symptom was chronic diarrhea (57.4\%). Seventy-two percent of the patients were malnourished. Giardia intestinalis was detected in 4, and Helicobacter
\end{abstract}

(c) 2018 S. Karger AG, Basel

E-Mail karger@karger.com www.karger.com/ddi pylori was confirmed in $8 / 45$ (17.7\%) patients. Non-celiac villous flatting was discovered in $15.5 \%$ of patients. Twelve patients were diagnosed as having immunodeficiency associated inflammatory bowel disease (IBD)-like colitis. Conclusions: PID may present with GI manifestations or develop during the course of the disease. Investigating immunodeficiency in patients with atypical Gl symptoms can provide an appropriate therapeutic option, and an improved quality of life, particularly in populations with a high rate of consanguinity.

(c) 2018 S. Karger AG, Basel

\section{Introduction}

Primary immunodeficiencies (PID) are a group of disorders that result from intrinsic impairments of the immune system [1]. Gut-associated lymphoid tissue is a barrier for immune system and plays an important role in maintaining the balance between active immunity and tolerance in the gut $[2,3]$. Also, secretory IgA controls the 
mucosal immune system and facilitates growth of normal gastrointestinal (GI) microbiota $[2,4,5]$. Dysregulation and defects in either humoral, cellular immunity or complement system may lead to an uncontrolled inflammatory process, and subsequently mucosal damage. Therefore, lymphoid tissue-rich GI tract is frequently affected in PID [4].

More than 150 genetically heterogeneous entities have come to be known as PID disorders $[1,2,6]$. These conditions usually manifest clinically as increased susceptibility to infections. However, clinical manifestations may be more subtle and surreptitious, and can present later in life, even during adulthood. Children with PID are prone to developing infectious, inflammatory, autoimmune diseases, and malignancies [7-11]. GI manifestations have been reported in approximately $5-50 \%$ of patients with PID $[2,12,13]$. GI manifestations in patients with PID may mimic other GI diseases, such as inflammatory bowel disease (IBD) and celiac disease; however, they differ in pathogenesis and response to the conventional treatment [4]. Thus, early recognition of GI manifestations of PIDs might alter the treatment strategy.

GI manifestations of childhood-onset PID are scarcely published in pediatric gastroenterology literature. The aim of this study was to analyze endoscopic, histopathological, and clinical findings among pediatric PID patients who had been evaluated for GI manifestations.

\section{Materials and Methods}

A total of 195 out of 425 children with an established diagnosis of PID after meticulous immunological and genetic investigations were presented to the division of the Pediatric Gastroenterology between 2005 and 2016. These children with PID, who had unremitting GI symptoms severe enough to require upper GI endoscopy and/or colonoscopy, were retrospectively analyzed. Patients were divided according to the updated classification for PID, established by the International Union of Immunological Societies Expert Committee [14].

Forty-seven patients with PID, and a total of 68 endoscopic examinations (45 upper GI tract endoscopy and 23 colonoscopy) were reviewed for this study. Histopathological samples from the GI tract were available in all patients for histopathological examination and a rapid urease test for Helicobacter pylori infection. Duodenal aspirate had also been collected for microscopic examination of giardia trophozoites in patients with growth failure. The same pathologist reinvestigated the endoscopic biopsy samples for this study. Demographic data, GI signs and symptoms, and growth parameters, assessed by using weight for height, height for age, and body mass index $\mathrm{Z}$ scores at diagnosis were noted. The study was approved by the local Ethics Committee of Marmara University School of Medicine. Informed written consents were obtained from parents or guardians of patients. The study protocol con- formed to the ethical guidelines of the 1975 Declaration of Helsinki, as reflected in a prior approval by the institution's human research committee.

\section{Results}

Of the 47 patients, $30(63.8 \%)$ were male, and the mean age of patients was $7.7 \pm 5$ years when they were consulted. The primary diagnosis of the patients are outlined in Table 1. The mean proportion of consanguineous marriages was $61.7 \%$ in the study group.

Both acute and chronic malnutrition were remarkable in the study group. Chronic malnutrition was found in $34 / 47$ (72\%) patients. Additionally, more than $60 \%$ (29/47) of the patients with PID had acute malnutrition, of which $20 \%(6 / 29)$ was severe.

Patients included in this study had variable GI symptoms such as chronic diarrhea with/without blood (57.4\%) and abdominal pain (53\%). GI manifestations were the presenting symptom in 24 of 47 patients (51\%), and evaluated mainly by a pediatrician or pediatric gastroenterologist at admission. Of the 24 patients, 13 (54\%) were referred during infancy (Table 2).

Endoscopic examination of the esophagus was normal in $57.4 \%$ of the patients. Congestion (49\%) and antral nodularity (16\%) were the most common endoscopic findings in the stomach. Duodenal ulcer was identified in 2 patients. H. pylori infection was confirmed with both rapid urease test and histopathological examination in 8 out of 45 $(17.7 \%)$ patients. Giardia intestinalis was detected in 4 patients by direct microscopic visualization of the parasite in the duodenal aspirates. Mucosal ulcer and hyperemia were commonly detected colonoscopic pathologies that were found in 43.4 and $30 \%$ of the patients, respectively.

Most of the patients (44.6\%) in the study group had predominant antibody deficiencies, specific diagnostic data of which are depicted in Table 1. Chronic diarrhea and growth retardation were the most common complaints. All patients in this group underwent gastroscopy, and villous atrophy was found in 6 . Two of them had nonceliac villous atrophy and the remaining 4 who had IgA deficiency were diagnosed as celiac disease. Colonoscopy was carried out in 10 of 21 patients with antibody deficiencies; and $50 \%$ of them had histopathological evidence of colitis (Table 3). Three of these patients demonstrated clinical and histopathological features of colitis mimicking IBD (Table 4).

Nine patients had combined immune deficiency with syndromic features (Table 1). The most common GI
46

Dig Dis 2019;37:45-52

DOI: $10.1159 / 000492569$
Akkelle/Tutar/Volkan/Sengul/Ozen/ Celikel/Ertem 
Table 1. Specific diagnoses of patients with primary immune deficiency

\begin{tabular}{lll}
\hline Group of immunodeficiency & Type of immunodeficiency & Patients, $n$ \\
\hline Predominantly antibody & CVID & 7 \\
deficiencies $(n=21)$ & Selective IgA deficiency & 8 \\
& IgG subclasses deficiency & 1 \\
& Hypogammaglobulinemia & 1 \\
\hline Combined immune deficiency & Agammaglobulinemia & 2 \\
with associated or syndromic & PIK3CD mutation & 2 \\
features $(n=9)$ & Ataxia telangiectasia & 2 \\
& Nijmegen breakage syndrome & 1 \\
\hline Immunodeficiency affecting & Bloom syndrome & 2 \\
cellular and humoral immunity $(n=5)$ & HIES & 2 \\
& ICF syndrome & 1 \\
\hline Congenital defects of phagocyte & THE syndrome & 1 \\
number and/or function $(n=5)$ & SCID & 2 \\
\hline Diseases of the immune & CSR defect & 1 \\
dysregulation $(n=3)$ & LRBA deficiency & 2 \\
\hline Complement deficiencies $(n=3)$ & Congenital neutropenia & 4 \\
\hline Undetermined $(n=1)$ & CGD & 1 \\
\hline & IL10RB deficiency & 1 \\
\hline I $n$ & IPEX & 2 \\
\hline
\end{tabular}

CVID, common variable immunodeficiency; PIK3CD, phosphatidylinositol-4,5-Bisphosphate 3-Kinase Catalytic Subunit Delta; HIES, hyper IgE syndrome; ICF, immunodeficiency with centromeric instability and facial anomalies; THE, trichohepatoenteric syndrome; SCID, severe combined immunodeficiency; CSR, class switch recombination defect; LRBA, lipopolysaccharide responsive beige-like anchor deficiency; CGD, chronic granulomatous disease; IPEX, immunodysregulation, polyendocrinopathy, enteropthy-X linked.

Table 2. The distribution of patients manifested with GI symptoms

\begin{tabular}{lll}
\hline Type of immunodeficiency & $\begin{array}{l}\text { Number of patients } \\
\text { presented with GI symptoms }\end{array}$ & $\begin{array}{l}\text { Number of patients } \\
\text { presented at infancy }\end{array}$ \\
\hline LRBA deficiency $(n=2)$ & 2 & 1 \\
Bloom syndrome $(n=2)$ & 1 & 0 \\
HIES $(n=2)$ & 2 & 1 \\
CVID $(n=7), n(\%)$ & $4(57)$ & 3 \\
Selective IgA deficiency $(n=8), n(\%)$ & $6(75)$ & 2 \\
IL10RB def. $(n=1)$ & 1 & 1 \\
CD55 def. $(n=3)$ & 3 & 1 \\
Hypogammaglobulinemia $(n=1)$ & 1 & 0 \\
Agammaglobulinemia $(n=2)$ & 1 & 1 \\
CD4 lymphopenia $(n=1)$ & 1 & 1 \\
THE syndrome $(n=1)$ & 1 & 1 \\
IPEX $(n=2)$ & 1 & 1 \\
\hline
\end{tabular}

LRBA, lipopolysaccharide responsive beige-like anchor deficiency; HIES, hyper IgE syndrome; CVID, common variable immunodeficiency; THE, trichohepatoenteric syndrome; IPEX, immunodysregulation, polyendocrinopathy, enteropthy-X linked. 
Table 3. Histopathological findings of endoscopic biopsies in PID patients with antibody deficiencies

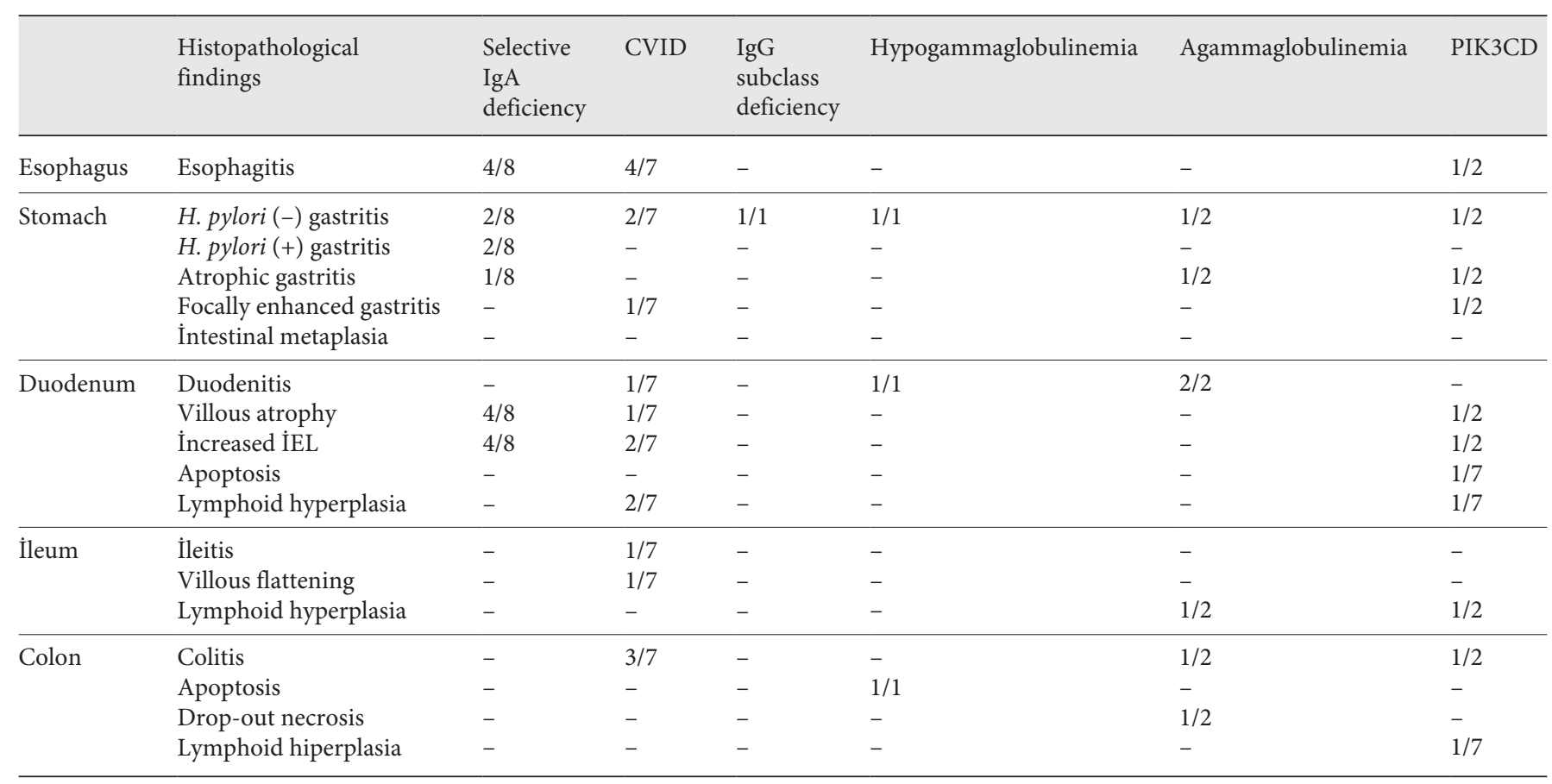

complaint was abdominal pain in this group. Upper GI endoscopy revealed esophageal varices in 3 patients with hyper-IgE syndrome, Bloom and ICF (Immunodeficiency with Centromeric instability and Facial anomalies) syndrome along with portal hypertension. One of the patients with Bloom syndrome, already diagnosed and treated for Wilm's tumor, was consulted for unrelenting abdominal pain and chronic diarrhea. Duodenal biopsies showed focal destruction of intestinal villi, increased intraepithelial $\mathrm{T}$ lymphocytes, and apoptosis. Lymphoid hyperplasia and cryptitis were the histopathologies observed in the colonic biopsies. The second patient with Bloom syndrome who was admitted to the hospital with severe obstructive symptoms underwent an abdominal surgery, and diffuse B cell lymphoma was diagnosed after histopathological examination of the jejunal specimen. Another patient with syndromic features was consulted with complaints of growth retardation and intractable diarrhea at 9 months of age. Multiple ulcerations were observed at sigmoid colon, and histopathological examination demonstrated vacuolar changes in epithelial cells and inflammation with increased eosinophils, suggesting colitis, mimicking IBD (Table 4). Subsequent genetic investigation revealed mutations in the SKIV2L gene and the diagnosis was TricoHepatoEnteric Syndrome.
Table 4. Primary immunodeficiencies presenting IBD-like phenotype and age at presentation

\begin{tabular}{ll}
\hline $\begin{array}{l}\text { Diagnosis of PID } \\
\text { (number of patients) }\end{array}$ & Age at presentation \\
\hline CD55 $(n=3)$ & 3,5 , and 16 years \\
G6PC3 $(n=2)$ & 10 and 16 years \\
CVID $(n=1)$ & 11 years \\
RAG2 deficiency (SCID; $n=1)$ & 9 years \\
Agammaglobulinemia $(n=1)$ & 15 years \\
PIK3CD $(n=1)$ & 7 years \\
IL10RB deficiency $(n=1)$ & 11 months \\
CD4 lymphopenia $(n=1)$ & 1 year \\
SKIV2L mutation $($ THE; $n=1)$ & 9 months
\end{tabular}

G6PC3, glucose-6-phosphatase catalytic subunit 3 deficiency; CVID, common variable immunodeficiency; RAG2, recombination activating gene 2; SCID, severe combined immunodeficiency; PIK3CD, phosphatidylinositol-4,5-Bisphosphate 3-Kinase Catalytic Subunit Delta; SKIV3L, superkiller viralicidic activity 2-like; THE, trichohepatoenteric syndrome.

In the study group, there were 5 patients with immunodeficiency affecting both cellular and humoral immunity (Table 1), and chronic diarrhea was the most common (80\%) manifestation. The histopathological findings of both upper GI tract and ileocolonic biopsies are listed in Table 5 . Two of the 5 patients who had already been
48

Dig Dis 2019;37:45-52

DOI: $10.1159 / 000492569$
Akkelle/Tutar/Volkan/Sengul/Ozen/ Celikel/Ertem 
Table 5. Histopathological findings in patients with combined immunodeficiency

$\begin{array}{llll}\text { Increased } & \text { Villous } & \text { Apoptosis } & \text { Lymphoid } \\ \text { intraepithelial } & \text { atrophy } & & \text { hyperplasia } \\ \text { T lymphocytes } & & \end{array}$

SCID (patient number 1: RAG 2 deficiency)

Upper GI tract endoscopy

Colonoscopy

SCID (patient number 2: RAG 2 deficiency)

Upper GI tract endoscopy

CSR defect (patient number 3)

Upper GI tract endoscopy

LRBA deficiency (patient number 4)

Upper GI tract endoscopy

Colonoscopy

LRBA deficiency (patient number 5)

Upper GI tract endoscopy

mocytes

SCID, severe combined immunodeficiency; RAG 2, recombination activating gene 2; CSR, class switch recombination defect; LRBA, lipopolysaccharide responsive beige-like anchor.
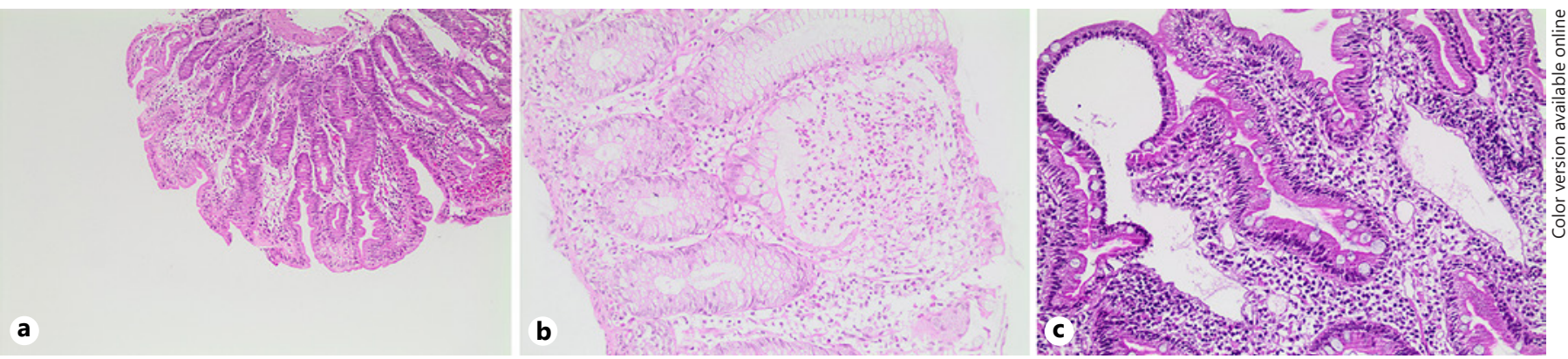

Fig. 1. Histopathological findings of endoscopic biopsies (H\&E staining). a Duodenal biopsy with villous blunting and increased apoptosis in crypts. b Colonic biopsy with crypt abscess and apoptosis at the basal crypts. c Duodenal biopsy with partial villous blunting and mucosal lacteal dilatation.

diagnosed with celiac disease were referred because of persistent diarrhea despite a gluten-free diet. Refractory villous atrophy, in association with scattered apoptotic cells in the duodenum and colon (Fig. 1a), prompted further immunological investigation. Ultimate diagnosis was achieved in these 2 patients after detecting mutations in RAG2 and LRBA genes.

In congenital defects of phagocyte number and/or function group, there were 4 patients with congenital neutropenia, and 3 patients with mutations in the G6PC3 gene (glucose-6-phosphatase catalytic subunit), resulting in G6PC3 deficiency associated with severe congenital neutropenia (Table 1). One of them presented with recurrent bouts of subtotal intestinal obstruction, and colonoscopy revealed severely inflamed stricture in the hepatic flexura. Eventually, the patient underwent hemicolectomy, which disclosed transmural inflammation consistent with IBD (Table 4). Another patient with G6PC3 deficiency was referred for evaluation of the intractable diarrhea associated with failure to thrive. Multiple erosions and ulcerations were observed in all colonic segments, which revealed apoptosis, increased lymphocytes, and plasma cells in the lamina propria, consistent with IBDlike colitis.

One patient, who was evaluated on account of intractable diarrhea, and recurrent perianal abscesses starting within the first months of life, underwent a colonoscopy. Colonic biopsies showed inflammatory cell infiltration with a preponderance of eosinophils, cryptitis, cryptic abscesses, and drop out necrosis, which were consistent 
with immunodeficiency-associated colitis (Fig. 1b). Subsequently, IL10R gene sequencing in this patient revealed a mutation, resulting in the deficiency of IL10RB that is characterized with immune dysregulation (Table 1). The patient received hematopoietic stem cell transplantation by the age of 14 months after which GI complaints resolved significantly.

Three of our patients who complained of chronic diarrhea, peripheral edema, and hypoalbuminemia were investigated by colonoscopy. Colonic biopsies showed scarce apoptotic bodies, reduced goblet cells, and moderate degree of inflammation, particularly increased lymphocytes and plasma cells, suggesting IBD. However, none of the patients responded to conventional immunosuppressive treatment, and one of them underwent surgery because of recurrent bouts of intestinal obstruction. The histopathological examination of transmural jejunal biopsies of the patient revealed the presence of lymphangiectasia (Fig. 1c). As a consequence, 3 patients were referred to the pediatric immunology for further immunological investigation because of immunosuppressive-resistant protein losing enteropathy. The genetic investigations disclosed deficiency of complement decayaccelerating factor (CD55), namely the CHAPLE syndrome (Table 1).

\section{Discussion}

PID are a heterogeneous group of inherited disorders, characterized by variable genetic immune defects. Since GI tract is the largest organ of the immune system, and is constantly exposed to antigens, GI symptoms may be the most prominent or sole manifestation of PID.

Growth retardation is an important consequence of all chronic diseases, which may affect both the prognosis of the disease and the quality of life. The rate of acute and chronic malnutrition in our study group was 60 and 75\%, respectively.

In the Eastern Europe, it has been reported that the incidence of $H$. pylori infection among children who underwent upper GI endoscopy was $40-45 \%[15,16]$. The overall rate of $H$. pylori infection was $17.7 \%$ in this cohort, and the infection was detected in $28 \%$ of the children with CVID. This rather lower rate of $H$. pylori infection among PID patients compared to the rate observed in immunecompetent children might be explained by the frequent use of antibiotics. This figure is similar to the previously published incidence in adult and pediatric CVID patients $[17,18]$.
Giardia is a common infectious cause of chronic diarrhea along with villous atrophy in PID, particularly in patients with IgA deficiency. Giardia trophozoites were detected in 4 patients with PID (agammaglobulinemia, hyper IgE syndrome, and 2 patients with IgA deficiency). Therefore, microscopic examination of the duodenal fluid aspirate for giardia trophocytes might be a simple and complementing test in patients who underwent a gastroscopy.

Patients with selective immunoglobulin A deficiency have a 10 - to 20 -fold increased risk of developing celiac disease. The prevalence of celiac disease in IgA-deficient people has been reported to be between 10 and 30\% [7]. Since IgG-based serological tests are not sufficiently reliable, and IgA-based tests are usually negative in these patients, serologic screening of celiac disease would be misleading in patients with PID. In this study, examination of duodenal biopsies revealed celiac disease in half of the patients with IgA deficiency. Flattening of the intestinal villi, resembling classical celiac disease is a common histopathological finding in PID $[17,19]$. Previously, intraepithelial lymphocytosis with villous atrophy had been demonstrated in $31-60 \%$ of patients with CVID [7, 17]. However, celiac disease-specific IgA and IgG antibodies are usually absent in those patients [20]. In this cohort, 11 patients (24.4\%) had villous atrophy in the duodenal mucosa, and 4 of them were diagnosed with celiac disease. Hence, the rate of non-celiac villous atrophy was $15.5 \%$ in this study group. Two of the patients with non-celiac villous flattening, who had not responded to a gluten-free diet during follow-up, was diagnosed with RAG 2 and LRBA deficiency after genetic analysis.

IBD is a chronic inflammatory disease caused by a dysregulated immune response to host intestinal microbiota [21]. Patients with PID have a higher risk of developing intestinal inflammation compared to the immune-competent individuals. In our cohort, colitis was found in $42.8 \%$ of the patients with CVID, and 1 out of 7 patients with CVID presented with Crohn-like clinical and histopathological features except for the absence of plasma cells in the lamina propria of the colon biopsies. IBD-like manifestations have been reported in nearly $4-6 \%$ of patients with CVID [7, 22, 23]. In our study population, 12 patients with different PID had presented with clinical features of enteropathy and/or colitis, and histopathological findings of the colonic and/or intestinal biopsies were similar to the patients with IBD as well.

Although IBD is a polygenic disorder, there is a diverse spectrum of rare genetic disorders, which mimic clinical and endoscopic features of IBD [24-29]. The rate of 
monogenic disorders presenting with IBD-like manifestations correlates inversely with the age at disease onset $[24,30,31]$. In our study group, 8 of 12 patients with IBDlike colitis were diagnosed with PID, with a defined genetic defect. Moreover, symptoms manifested before 10 years of age in 6 of them corresponded to the definition of early onset IBD.

In conclusion, GI manifestations due to PID are common, often associated with high morbidity. Usually, they do not respond to conventional treatments. Therefore, pediatric gastroenterologists are in a position to assist in recognition and diagnosis of patients with PID. In coun- tries with a high percentage of consanguineous marriages such as ours, PID should be suspected in every child with unrelenting GI symptoms, particularly starting at early ages. In recent years, advancement and availability of genetic investigations have enabled the discovery of newly recognized PID, and allowed the early initiation of more specific therapies that may improve the quality of life.

\section{Disclosure Statement}

The authors declare that there is no conflict of interest.

\section{References}

1 Holland SM, Bellanti JA: Immune deficiency disorders; in Bellanti JA (ed): Immunology 4: Clinical Applications in Health and Disease. Washington, I Care Press, 2012, pp 559-639.

2 Kobrynski LJ, Mayer L: Diagnosis and treatment of primary immunodeficiency disease in patients with gastrointestinal symptoms. Clin Immunol 2011;139:238-248.

3 Spahn TW, Kucharzik T: Modulating the intestinal immune system: the role of lymphotoxin and GALT organs. Gut 2004;53:456465.

4 Kadaoui KA, Corthesy B: Secretory IgA modiates bacterial translocation to dendritic cells in mouse Peyer's patches with restriction to mucosal compartment. J Immunol 2007;179: 7751-7757.

5 Bollinger RR, Everett ML, Palestrant D, Love SD, Lin SS, Parker W: Human secretory immunoglobulin A may contribute to biofilm formation in the gut. Immunology 2003;109: 315-327.

6 Geha RS, Notarangelo LD, Casanova JL, Chapel H, Conley ME, Fischer A, Hammarström L, Nonoyama S, Ochs HD, Puck JM, Roifman C, Seger R, Wedgwood J; International Union of Immunological Societies Primary Immunodeficiency Diseases Classification Committee: Primary immunodeficiency diseases: an update from the International Union of Immunological Societies Primary Immunodeficiency Diseases Classification Committee. J Allergy Clin Immunol 2007:120:776794.

7 Guerrerio AL, Frischmeyer-Guerrerio PA, Lederman HM, Oliva-Hemker M: Recognizing gastrointestinal and hepatic manifestations of primary immunodeficiency diseases. J Pediatr Gastroenterol Nutr 2010;51:548-555.

8 Uzzan M, Ko HM, Mehandru S, Cunningham-Rundles C: Gastrointestinal disorders associated with common variable immune deficiency (CVID) and chronic granulomatous disease (CGD). Curr Gastroenterol Rep 2016;18:17.
9 Quinti I, Soresina A, Spadaro G, Martino S, Donnanno S, Agostini C, Claudio P, Franco D, Maria Pesce A, Borghese F, Guerra A, Rondelli R, Plebani A: Long-term follow-up and outcome of a large cohort of patients with common variable immunodeficiency. J Clin Immunol 2007;27:308-316.

10 Al-Muhsen SZ: Gastrointestinal and hepatic manifestations of primary immune deficiency diseases. Saudi J Gastroenterol 2010;16:6674.

11 Fischer A, Provot J, Jais JP, Alcais A, Mahlaoui $\mathrm{N}$ : Autoimmune and inflammatory manifestations occur frequently in patients with primary immunodeficiencies. J Allergy Clin Immunol 2017;140:1388-1393.

12 Lai Ping So A, Mayer L: Gastrointestinal manifestations of primary immunodeficiency disorders. Semin Gastrointest Dis 1997;8:22-32.

13 Wood P, Stanworth S, Burton J, Jones A, Peckham DG, Green T, Hyde C, Chapel H: Recognition, clinical diagnosis and management of patients with primary antibody deficiencies: a systematic review. Clin Exp Immunol 2007; 149:410-423.

14 Picard C, Al-Herz W, Bousfiha A, Casanova JL, Chatila T, Conley ME, CunninghamRundles C, Etzioni A, Holland SM, Klein C, Nonoyama S, Ochs HD, Oksenhendler E, Puck JM, Sullivan KE, Tang ML, Franco JL, Gaspar HB: Primary immunodeficiency diseases: an update on the classification from the international union of immunological societies expert committee for primary immunodeficiency 2015. J Clin Immunol 2015;35:696726.

15 Ozbey G, Dogan Y, Demiroren K, Ozercan IH: Prevalence of Helicobacter pylori in children in eastern Turkey and molecular typing of isolates. Braz J Microbiol 2015;46:505-511.

16 Koca T, Serdaroglu F, Dereci S, Akcam M: Peptic ulcers and erosions in children at a pediatric unit in Turkey. Indian Pediatr 2016;53: 692-694.

17 Malamut G, Verkarre V, Suarez F, Viallard JF, Lascaux AS, Cosnes J, Bouhnik Y, Lambotte O,
Bechade D, Ziol M, Lavergne A, Hermine O, Cerf-Bensussan N, Cellier C: The enteropathy associated with common variable immunodeficiency: the delineated frontiers with celiac disease. Am J Gastroenterol 2010;105:2262-2275.

18 Lougaris V, Ravelli A, Villanacci V, Salemme M, Soresina A, Fuoti M, Lanzarotto F, Lanzini A, Plebani A, Bassotti G: Gastrointestinal pathologic abnormalities in pediatric and adult-onset common variable immunodeficiency. Dig Dis Sci 2015;60:2384-2389.

19 Kalha I, Sellin JH: Common variable immunodeficiency and the gastrointestinal tract. Curr Gastroenterol Rep 2004;6:377-383.

20 Agarwal S, Mayer L: Diagnosis and tretment of gastrointestinal disorders in patients with primary immunodeficiency. Clin Gastroenterol Hepatol 2013;11:1050-1063.

21 Arkwright PD, Abinun M, Cant AJ: Autoimmunity in human primary immunodeficiency diseases. Blood 2002;99:2694-2702.

22 Hermaszewski RA, Webster AD: Primary hypogammaglobulinaemia: a survey of clinical manifestations and complications. Q J Med 1993;86:31-42.

23 Cunningham-Rundles C, Bodian C: Common variable immunodeficiency: clinical and immunological features of 248 patients. Clin Immunol 1999;92:34-48.

24 Kammermeier J, Dziubak R, Pescarin M, Drury S, Godwin H, Reeve K, Chadokufa S, Huggett B, Sider S, James C, Acton N, Cernat E, Gasparetto M, Noble-Jamieson G, Kiparissi F, Elawad M, Beales PL, Sebire NJ, Gilmour K, Uhlig HH, Bacchelli C, Shah N: Phenotypic and genotypic characterisation of inflammatory bowel disease presenting before the age of 2 years. J Crohns Colitis 2017;11: 60-69.

25 Fabre A, Breton A, Coste ME, Colomb V, Dubern B, Lachaux A, Lemale J, Mancini J, Marinier E, Martinez-Vinson C, Peretti N, Perry A, Roquelaure B, Venaille A, Sarles J, Goulet O, Badens C: Syndromic (phenotypic) diarrhoea of infancy/tricho-hepato-enteric syndrome. Arch Dis Child 2014;99:35-38. 
26 Ozen A, Comrie WA, Ardy RC, Domínguez Conde C, Dalgic B, Beser ÖF, Morawski AR, Karakoc-Aydiner E, Tutar E, Baris S, Ozcay F, Serwas NK, Zhang Y, Matthews HF, Pittaluga S, Folio LR, Unlusoy Aksu A, McElwee JJ, Krolo A, Kiykim A, Baris Z, Gulsan M, Ogulur I, Snapper SB, Houwen RHJ, Leavis HL, Ertem D, Kain R, Sari S, Erkan T, Su HC, Boztug K, Lenardo MJ: CD55 deficiency, earlyonset protein-losing enteropathy, and thrombosis. N Engl J Med 2017;377:52-61.

27 Bindl L, Torgerson T, Perroni L, Youssef N, Ochs HD, Goulet O, Ruemmele FM: Successful use of the new immune-suppressor sirolimus in IPEX (immune dysregulation, polyen- docrinopathy, enteropathy, X-linked syndrome). J Pediatr 2005:147:256-259.

28 Bégin P, Petey N, Mueller P, Rasquin A, Sirard A, Klein C, Haddad E, Drouin É, Le Deist F: Inflammatory bowel disease and $\mathrm{T}$ cell lymphopenia in G6PC3 deficiency. J Clin Immunol 2013:33:520-525.

29 Kiykim A, Baris S, Karakoc-Aydiner E, Ozen AO, Ogulur I, Bozkurt S, Ataizi CC, Boztug K, Barlan IB: G6PC3 deficiency: primary immune deficiency beyond just neutropenia. J Pediatr Hematol Oncol 2015:37:616-622.

30 Glocker EO, Kotlarz D, Boztug K, Gertz EM, Schäffer AA, Noyan F, Perro M, Diestelhorst J, Allroth A, Murugan D, Hätscher N, Pfeif- er D, Sykora KW, Sauer M, Kreipe H, Lacher M, Nustede R, Woellner C, Baumann U, Salzer U, Koletzko S, Shah N, Segal AW, Sauerbrey A, Buderus S, Snapper SB, Grimbacher B, Klein C: Inflammatory bowel disease and mutations affecting the interleukin-10 receptor. N Engl J Med 2009;361: 2033-2045.

31 Uhlig HH, Schwerd T, Koletzko S, Shah N, Kammermeier J, Elkadri A, Ouahed J, Wilson DC, Travis SP, Turner D, Klein C, Snapper SB, Muise AM: The diagnostic approach to monogenic very early onset inflammatory bowel disease. Gastroenterology 2014;147: 990-1007. 